01

\title{
Гистерезис в поведении трехмерной упорядоченной джозефсоновской среды в магнитном поле при немалых значениях параметра пиннинга
}

\author{
(C) М.A. Зеликман \\ Санкт-Петербургский государственный политехнический университет Петра Великого, \\ 195251 Санкт-Петербург, Россия \\ e-mail: marzelik@mail.ru
}

(Поступило в Редакцию 13 декабря 2017 г. В окончательной редакции 26 марта 2018 г.)

На базе подхода, основанного на анализе непрерывного видоизменения конфигурации, протекающего в направлении уменьшения потенциала Гиббса, рассчитана кривая намагничивания трехмерной упорядоченной джозефсоновской среды при циклическом изменении внешнего магнитного поля для немалых значений параметра пиннинга. Показано, что крайние области петли гистерезиса всегда лежат на основной кривой намагничивания. Исследованы изменения вихревых последовательностей, а также профиля магнитного поля в образце при циклическом изменении внешнего поля. Показано, что рельеф магнитного поля в образце состоит из двух линейных участков - возрастающего и убывающего - с одинаковыми по модулю значениями крутизны.

DOI: 10.21883/JTF.2018.11.46620.2597

\section{Введение}

Вид кривой намагничивания материала определяется процессами, происходящими при проникновении магнитного поля в образец. Представляет интерес исследование кривой намагничивания высокотемпературных гранулированных сверхпроводников (ВТСП). Их поведение в магнитном поле заметно отличается от поведения обычных сверхпроводников. В массивных сверхпроводниках второго рода, помещенных во внешнее магнитное поле $H_{e}$, начиная со значения $H_{e}=H_{C 1}$ становится энергетически выгодным переход в смешанное состояние, когда магнитное поле частично проникает в толщу сверхпроводника в виде вихревых нитей. В то же время уравнения электродинамики формально допускают, вплоть до термодинамического критического поля $H_{C}$, равновесные решения мейсснеровского типа с проникновением поля в тонкую приповерхностную область. Таким образом, существует возможность задержки проникновения вихрей в сверхпроводник для полей $H_{e}>H_{C 1}$. С точки зрения термодинамики это явление представляет собой „перегрев“ мейсснеровского состояния. Максимальное поле $H_{S 1}$, до которого возможен этот „перегрев“, определяется как граница устойчивости метастабильного мейсснеровского состояния по отношению к малым флуктуациям $[1,2]$.

Аналогичная картина имеет место и в ВТСП. Однако физика происходящих в них процессов заметно отличается от того, что происходит в обычных сверхпроводниках. Важнейшую роль играет тот факт, что ВТСП состоят из сверхпроводящих гранул, в точках соприкосновения которых друг с другом возникают джозефсоновские контакты. Поэтому иной вид имеют вихри, иной физике соответствуют критические поля.
В частности, характерные величины полей столь малы, что речь не может идти о переходе гранул в нормальное состояние (поэтому поле $H_{S 2}$, до которого существует мейсснеровское решение, отличается от термодинамического поля $H_{C}$ ). Вихри располагаются не в отдельных гранулах, а вовлекают в себя большое количество их, при этом токи проходят через джозефсоновские контакты между гранулами. Достаточно вспомнить, что такой контакт является нелинейным элементом, чтобы представить себе сложность возникающей картины. Добавим к этому еще и зацепление (пиннинг) вихрей на пустотах между гранулами. Все эти моменты делают исследование поведения ВТСП во внешнем магнитном поле достаточно сложной задачей, требующей специального подхода.

В работах $[3,4]$ предложена простая модель гранулированного ВТСП, представляющая собой кубическую решетку, состоящую из сверхпроводящих проводов, каждый из которых содержит один джозефсоновский контакт. На базе условий квантования флюксоида в ячейках этой среды получена система уравнений для расчета структуры возможных токовых состояний. Как показали расчеты [3-5], такой модели, которую принято называть трехмерной упорядоченной джозефсоновской средой, присущи все свойства, характерные для сверхпроводников во внешнем магнитном поле: мейсснеровские экранирующие токи, взаимодействующие друг с другом вихри, набор характерных магнитных полей и т.п., причем даже количественные соотношения аналогичны имеющим место в обычных и высокотемпературных сверхпроводниках. Поэтому использование этой модели целесообразно при анализе процессов, происходящих в реальных ВТСП. 
В работе [5] рассмотрена устойчивость мейсснеровского состояния трехмерной джозефсоновской среды относительно малых флуктуаций скачков фазы на контактах. Показано, что величина $H_{S 1} / H_{S 2}$ растет с увеличением параметра пиннинга $I$ и находится в пределах от 0.84 до 1 , что согласуется с результатами, полученными для обычных сверхпроводников второго рода.

В сверхпроводниках второго рода предел мейсснеровского состояния определяется равенством энергий нормального и сверхпроводящего состояний с учетом энергии экранирующих токов и равен $H_{C}$. Если внешнее поле больше $H_{C}$, то образец переходит в нормальное состояние. В рассматриваемом случае джозефсоновской среды эти соображения неприменимы. Что же будет происходить, когда внешнее поле превысит величину $H_{S 2}$ и мейсснеровское состояние невозможно? В работах $[6,7]$ предложен подход, основанный на анализе непрерывного видоизменения конфигурации, протекающего в направлении уменьшения потенциала Гиббса. Расчет [7] показал, что характер вихревой картины определяется величиной так называемого параметра пиннинга $I$, определение которого будет дано далее. При значениях $I$, больших некоторого критического (находящегося в диапазоне 0.7-0.8), вихри с ростом поля постепенно продвигаются от границы внутрь, а магнитное поле в глубине образца остается равным нулю. Если же $I<I_{C}$, то такая приграничная структура может существовать лишь до значения внешнего поля $H_{\max }(I)$. При $H_{e}>H_{\max }$ магнитное поле проникает внутрь образца на всю его глубину. В работах $[8,9]$ детально анализируются основная кривая намагничивания, а также петля гистерезиса для намагниченности трехмерной упорядоченной джозефсоновской среды в случае малых значений параметра пиннинга $(I=0.9)$.

Целью настоящей работы является исследование гистерезиса при циклическом изменении магнитного поля для случая $I>I_{C}$. Для этого случая основная кривая намагничивания, получаемая при монотонном увеличении внешнего магнитного поля $H_{e}$, исследована в [7]. Рассмотрим теперь циклическое изменение внешнего магнитного поля. Сначала будем увеличивать $H_{e}$ от нуля. До значения поля $H_{S 1}$ реализуется мейсснеровское решение. При $H_{S 1}<H_{e}<H_{\max }$ возникает приграничная последовательность вихрей, полностью компенсирующая внешнее поле в глубине контакта. Дойдя до некоторого значения поля $H_{a}$, начнем отслеживать развитие ситуации при уменьшении $H_{e}$. Достигнув значения $-H_{a}$, снова начнем увеличивать поле. Таким способом будет исследован весь цикл.

\section{Мейсснеровская конфигурация}

Рассмотрим сначала токовую конфигурацию мейсснеровского типа, т.е. когда вдоль поверхности образца текут параллельные ей токи, замыкающиеся на бесконечности (рис. 1). Сила тока, текущего в граничном

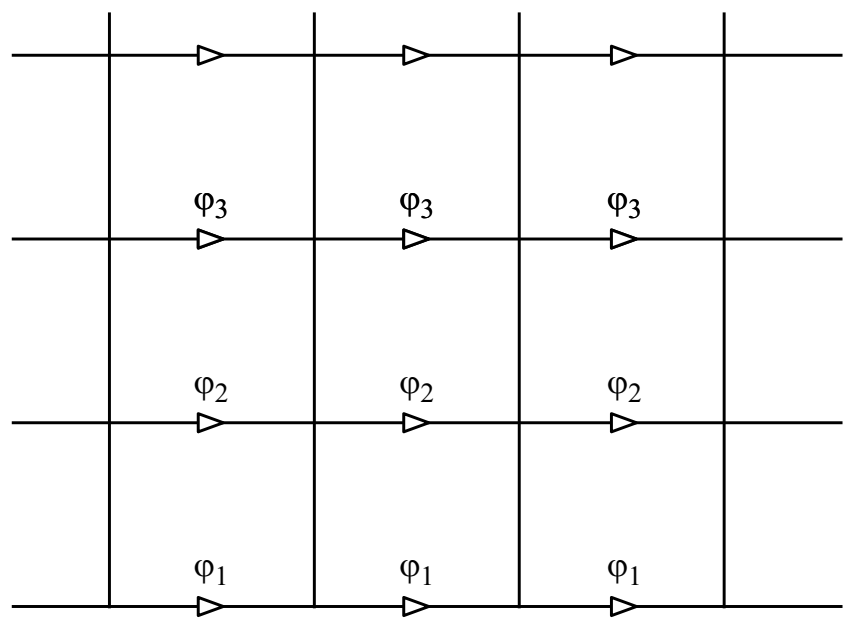

Рис. 1. Конфигурация мейсснеровского типа.

слое, равна $J_{1}=J_{C} \sin \varphi_{1}$. По мере углубления в образец имеем $J_{m}=J_{C} \sin \varphi_{m}$ для $m=2,3 \ldots$ В глубине образца токи равны нулю, что является одним из граничных условий.

В каждой из ячеек выполняется условие квантования флюксоида [3], согласно которому сумма скачков фазы по замкнутому контуру с джозефсоновскими контактами должна равняться нормированному магнитному потоку через контур, т. е.

$$
2 \pi \Phi_{m} / \Phi_{0}+\sum_{k} \varphi_{k}^{(m)}=0,
$$

где $\sum_{k} \varphi_{k}^{(m)}$ - сумма скачков фазы на джозефсоновских контактах $m$-й ячейки с учетом знака, $\Phi_{0}-$ квант магнитного потока, $\Phi_{m}-$ полный магнитный поток через $m$-ю ячейку. Обычно в правой части условия квантования флюксоида пишется член $2 \pi N_{m}$, где $N_{m}$ целое число, равное величине флюксоида в $m$-й ячейке. При этом скачки фазы по модулю не должны превышать величины $\pi$. Мы же не будем накладывать на них никаких ограничений. Найдя решение, мы сможем вычислить $N_{m}$, пересчитав фазы, как будет показано далее.

Для рассматриваемой мейсснеровской конфигурации (1) принимает вид

$$
2 \pi \Phi_{m} / \Phi_{0}+\varphi_{m}-\varphi_{m+1}=0,
$$

а магнитный поток $\Phi_{m}$ описывается выражением [3]

$$
\begin{aligned}
\Phi_{m}= & \mu_{0}(a-2 \delta) \int_{\delta}^{a-\delta} H(r) d r=\mu_{0}(a-2 \delta)^{2} \\
& \times\left(\sum_{k=1}^{m} \frac{J_{k}}{a}+\frac{b}{a}\left(J_{m-1}-J_{m}\right)-H_{e}\right),
\end{aligned}
$$

где параметр $b=\frac{1}{2 \pi(1-2 \delta / a)} \ln \frac{1-\exp (-2 \pi(1-\delta / a))}{1-\exp (-2 \pi \delta / a)}$. Далее мы будем рассматривать такие значения $I$ и $b$, что 
$I b \ll 1$, где $I-$ параметр пиннинга, определяемый выражением (4). Тогда после подстановки (3) в (1) появляется возможность пренебречь вторым членом в (3). Отметим, что такое рассмотрение справедливо также для искусственной структуры, созданной из сверхпроводящих нитей, склеенных друг с другом по всей длине, так что джозефсоновскими контактами являются поверхности соединения нитей. Внешнее поле направлено вдоль нитей. Поперечное сечение структуры должно представлять собой квадратную решетку, при этом форма ячейки не обязана быть квадратной, в частности, она может быть образована нитями круглого сечения. Легко показать, что в этой ситуации поле в ячейках однородно и $b=0$.

Подставив (3) в (2) для различных $m$ и вычтя из уравнения для $(m-1)$-й ячейки уравнение для $m$-й, получим следующую систему разностных уравнений:

$$
\varphi_{m+1}-2 \varphi_{m}+\varphi_{m-1}=I \sin \varphi_{m} \quad(m \geq 2),
$$

где $I=2 \pi \mu_{0}(a-2 \delta)^{2} J_{C} / a \Phi_{0}-$ так называемый параметр пиннинга.

В качестве граничных условий к (4) используем стремление к нулю $\varphi_{m}$ при стремлении $m$ к бесконечности, а также условие (1) для $m=1$, имеющее вид

$$
\varphi_{1}-\varphi_{2}=-I \sin \varphi_{1}+2 \pi h,
$$

где $h=H_{e} / H_{0}$ - нормированная напряженность внешнего поля, $H_{0}=\Phi_{0} / \mu_{0} S-$ значение внешнего поля, при котором через каждую ячейку площадью $S=(a-2 \delta)^{2}$ проходит 1 квант магнитного потока $\Phi_{0}$.

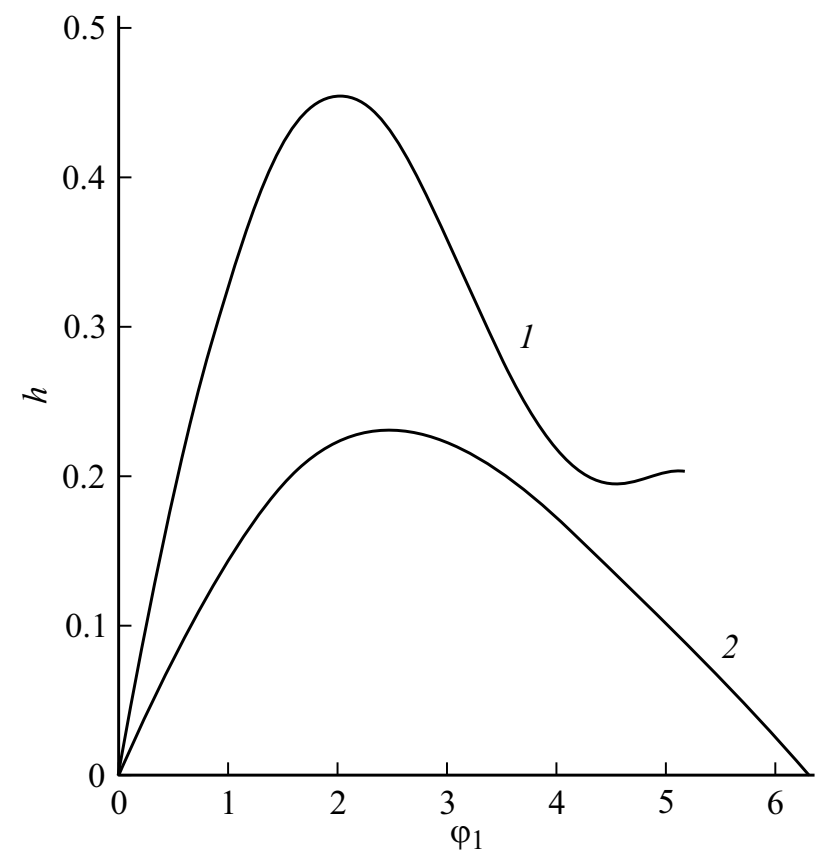

Рис. 2. Зависимость нормированной напряженности внешнего магнитного поля от скачка фазы на участке, ближайшем к границе, для $I=1.6-$ кривая 1 и $I=0.5-$ кривая 2 .
Найдем точное решение системы (4). Представим ее в виде рекуррентного закона

$$
\varphi_{m+1}=2 \varphi_{m}-\varphi_{m-1}+I \sin \varphi_{m} \quad(m \geq 2) .
$$

Задаваясь значениями $\varphi_{1}$, будем численно искать соответствующие им возможные значения $\varphi_{2}$, при которых существует решение в виде монотонно убывающей с ростом $m$ комбинации значений $\varphi_{m}$. Из уравнения (5) найдем соответствующее полученному решению значение нормированной напряженности внешнего магнитного поля $h$. На рис. 2 приведены полученные графики зависимости $h\left(\varphi_{1}\right)$ для некоторых значений $I$. Начальный участок кривой до максимума соответствует мейсснеровскому режиму. Максимально возможное значение $h_{S 2}$ соответствует величине магнитного поля $H_{S 2}$, выше которой мейсснеровское решение отсутствует. Развитие ситуации при дальнейшем увеличении поля будем исследовать на базе анализа потенциала Гиббса системы.

\section{Методика вычислений}

В работе [5] показано, что флуктуации, относительно которых мейсснеровская конфигурация неустойчива, представляют собой быстро затухающие вглубь (на нескольких слоях) периодически повторяющиеся знакопеременные структуры шириной в 1 ячейку. Это дает основания предположить, что в процессе развития неустойчивости распределение скачков фазы будет сохранять такую же геометрию (рис. 3). Равенство модулей и противоположность знаков скачков фазы в соседних вертикальных участках следует из периодичности конфигурации и условия непрерывности токов в узлах.

Будем рассматривать образец конечной длины $2 N+1$ ячеек. Пусть распределение фаз симметрично относительно центральной плоскости образца, как показано на рис. 3.

Потенциал Гиббса такой конфигурации высотой в $1 \mathrm{~m}$ записывается в виде [5]

$$
G=E_{J}+E_{H}-\int B H d V
$$

где $E_{J}$ и $E_{H}-$ джозефсоновская и магнитная энергии единицы длины (вдоль $\left.H_{e}\right)$, равные

$$
\begin{gathered}
E_{J}=\frac{E_{C}}{a} \sum_{k}\left(1-\cos \psi_{k}\right)=I E_{0} \sum_{k}\left(1-\cos \psi_{k}\right) . \\
E_{H}=\sum_{i} \frac{B_{i}^{2}}{2 \mu_{0}} V_{i}=E_{0} \frac{1}{2} \sum_{i}\left(\sum_{l} \psi_{i l}\right)^{2},
\end{gathered}
$$

где $E_{C}=\Phi_{0} J_{C} / 2 \pi-$ энергия джозефсоновского контакта, $B_{i}=\Phi_{i} / S$ - магнитная индукция в $i$-й ячейке, $B_{i}^{2} / 2 \mu_{0}$ - плотность магнитной энергии в $i$-й ячейке, $V_{i}=S$ - объем единицы длины, $E_{0}=\Phi_{0}^{2} / 4 \pi^{2} \mu_{0} S-$ нормировочная постоянная. Суммирование по $k$ в (8) 


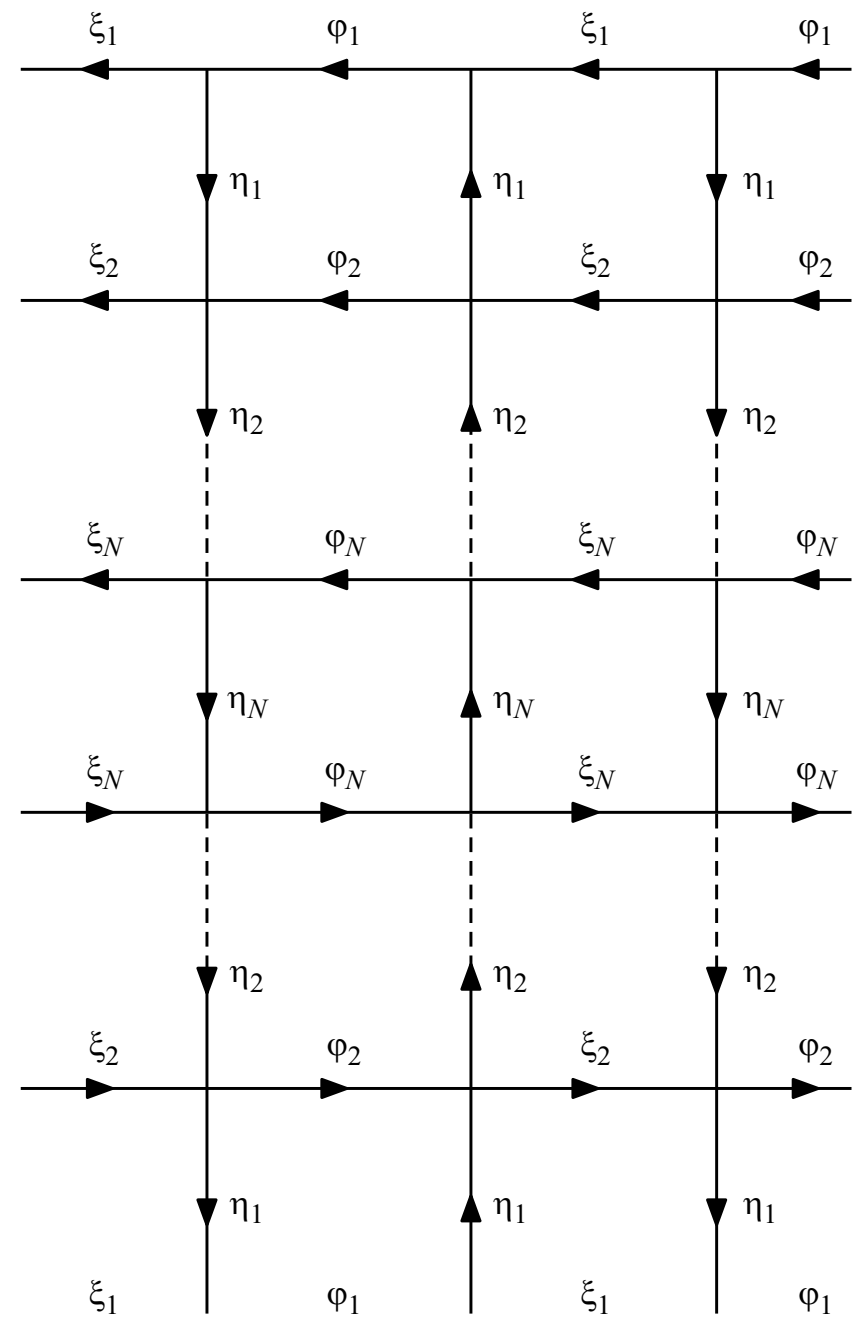

Рис. 3. Распределение скачков фазы в рассматриваемой конфигурации.

ведется по всем джозефсоновским контактам, по $i$ в (9) - по всем ячейкам, по $l$ во внутренней сумме в (9) - по всем 4-м контактам $i$-й ячейки (с учетом знака). Обозначение $\psi_{m}$ введено для любого скачка фазы с индексом $m$. При выводе (9) мы воспользовались условием квантования флюксоида (1).

Как известно, в рассматриваемой геометрии термодинамическая напряженность магнитного поля $H$ во всех точках одна и та же и равна напряженности внешнего поля $H_{e}$, поэтому последний член в (7) можно записать в виде

$$
H_{e} \sum_{i} \Phi_{i}=\frac{H_{e} \Phi_{0}}{2 \pi} \sum_{i} \sum_{l} \psi_{i l}=\frac{H_{e} \Phi_{0}}{2 \pi} \sum_{n} \psi_{1 n},
$$

где $\psi_{i l}-$ скачок фазы в $l$-м контакте $i$-й ячейки, а сумма по $n$ берется по всем контактам граничных рядов. При выводе (10) мы учли, что при суммировании по всем ячейкам все значения $\psi_{i l}$ для контактов внутри конфигурации входят в сумму дважды, причем с разны- ми знаками, поэтому остается только суммирование по граничным контактам.

С учетом вышесказанного выражение для потенциала Гиббса принимает вид

$$
\begin{aligned}
& G=E_{0}\left(\sum_{k=1}^{N-1}\left(\varphi_{k}-\varphi_{k+1}+2 \eta_{k}\right)^{2}+\sum_{k=1}^{N-1}\left(\xi_{k}-\xi_{k+1}+2 \eta_{k}\right)^{2}\right. \\
& +2\left(\varphi_{N}+\eta_{N}\right)^{2}+2\left(\xi_{N}-\eta_{N}\right)^{2}+2 I \sum_{k=1}^{N}\left(4-\cos \varphi_{k}-\cos \xi_{k}\right. \\
& \left.\left.-2 \cos \eta_{k}\right)-2 I\left(1-\cos \eta_{N}\right)-4 \pi h\left(\varphi_{1}+\xi_{1}\right)\right) .
\end{aligned}
$$

Далее будем измерять $G$ в единицах $E_{0}$. Будем трактовать величину $G$ как высоту (или потенциальную энергию) горного рельефа, заданного на многомерном множестве координат $\varphi_{i}, \xi_{i}$. Установившаяся при какомлибо значении внешнего поля $h$ мейсснеровская конфигурация (набор координат) соответствует минимуму энергии (впадине) в рельефе. С ростом $h$ рельеф постепенно изменяется, мейсснеровская конфигурация слегка смещается, но ей продолжает соответствовать локальный минимум. При достижении магнитным полем значения $h_{s}$ этот минимум становится седловой точкой. Если теперь система „сделает шаг“ в соответствующем направлении, т.е. получит нужную флуктуацию, то она окажется на склоне рельефа. Дальнейшее изменение формы этой конфигурации можно рассматривать как „скатывание“ по рельефу с уменышением „потенциальной энергии“, в результате которого система окажется в другом минимуме, в котором конфигурация уже не будет мейсснеровской. Пусть этот процесс происходит как результат большого количества маленьких шагов. Логично предположить, что в каждой „точке“ скатывание происходит вдоль линии наибыстрейшего спуска, т.е. вдоль градиента функции $G$. Это значит, что при каждом шаге все „координаты“ (т.е. $\left.\varphi_{i}, \xi_{i}\right)$ получают приращения, пропорциональные соответствующей проекции градиента: $\Delta \varphi_{i}=-\frac{\partial G}{\partial \psi_{i}} \delta$, где $\delta>0$ - малый постоянный множитель, задающий величину шага. Далее вычисляем все $\frac{\partial G}{\partial \psi_{i}}$ в новой точке, т.е. при новых значениях $\psi_{i}$, и производим следующий шаг. Эта процедура повторяется до тех пор, пока не придем к новой устойчивой конфигурации, находящейся в минимуме рельефа. Этим завершается первый этап расчета.

На втором этапе анализируется развитие ситуации при дальнейшем увеличении магнитного поля. Если $h$ увеличится на какую-то малую величину, то это приведет к некоторому видоизменению рельефа, в результате чего конфигурация, соответствовавшая минимуму прежнего рельефа, теперь окажется на склоне нового и „скатится“ в новый минимум. Последовательно увеличивая $h$, можно исследовать распределение скачков фазы $\varphi_{i}, \xi_{i}$, а значит, токов и магнитных полей, во всем диапазоне изменения внешнего поля. 
Перед тем, как записать выражения для $\frac{\partial G}{\partial \psi_{i}}$, учтем условия непрерывности токов в узлах. Последовательно записывая эти условия в узлах каждого ряда, начиная от граничного, получим

$$
\sin \eta_{k}=\sum_{i=1}^{k}\left(\sin \varphi_{i}-\sin \xi_{i}\right) \quad(k=1,2, \ldots N),
$$

откуда можно выразить значения $\eta_{k}$ в виде функций от $\varphi_{i}$ и $\xi_{i}$ :

$$
\eta_{k}=\arcsin \sum_{i=1}^{k}\left(\sin \varphi_{i}-\sin \xi_{i}\right)
$$

Тогда выражения для „проекций градиента“ $G$ по осям $\varphi_{i}$ принимают вид

$$
\begin{aligned}
& \partial G / \partial \varphi_{i}=2\left(2 \varphi_{i}-\varphi_{i-1}+\varphi_{i+1}+2\left(\eta_{i}-\eta_{i-1}\right)+I \sin \varphi_{i}\right. \\
& +2 \cos \varphi_{i} \sum_{k=i}^{N-1} \frac{\varphi_{k}-\varphi_{k+1}-\xi_{k}+\xi_{k+1}+4 \eta_{k}+I \sin \eta_{k}}{\cos \eta_{k}} \\
& \left.+\frac{\cos \varphi_{i}}{\cos \eta_{N}}\left(2 \varphi_{N}-2 \xi_{N}+4 \eta_{N}+I \sin \eta_{N}\right)\right) \\
& \partial G / \partial \varphi_{1}=2\left(-2 \pi h+\varphi_{1}-\varphi_{2}+2 \eta_{1}+I \sin \varphi_{1}\right. \\
& +2 \cos \varphi_{1} \sum_{k=1}^{N-1} \frac{\varphi_{k}-\varphi_{k+1}-\xi_{k}+\xi_{k+1}+4 \eta_{k}+I \sin \eta_{k}}{\cos \eta_{k}} \\
& \left.+\frac{\cos \varphi_{N}}{\cos \eta_{N}}\left(2 \varphi_{N}-2 \xi_{N}+4 \eta_{N}+I \sin \eta_{N}\right)\right) . \\
& \left.+\frac{\cos \varphi_{1}}{\cos \eta_{N}}\left(2 \varphi_{N}-2 \xi_{N}+4 \eta_{N}+I \sin \eta_{N}\right)\right) \\
& \partial G / \partial \varphi_{N+1}=2\left(3 \varphi_{N}-\varphi_{N-1}+2\left(\eta_{N}-\eta_{N+1}\right)+I \sin \varphi_{N}\right.
\end{aligned}
$$

Производные по $\xi_{i}$ можно получить из выражений (14)-(16), меняя местами все $\varphi_{i}$ и $\xi_{i}$ и поменяв везде знак у $\eta_{i}$ на обратный.

На рис. 4 приведены полученные в [5] графики зависимостей от $I$ величины $h_{S 1} / h_{S 2}$, а также $\varphi_{1 \text { min }}$, соответствующей значению $h_{S 1}$. В качестве начальной точки алгоритма возьмем мейсснеровскую конфигурацию при внешнем поле, немного превышающем $h_{S 1}$. Для этого рассчитаем мейсснеровскую конфигурацию по алгоритму (6) для начального значения, чуть большего $\varphi_{1 \min }$ для выбранного I. Далее дадим величинам $\varphi_{k}$ и $\xi_{k}$ для малых $k$ небольшие приращения, соответствующие неустойчивой флуктуации [5] при внешнем

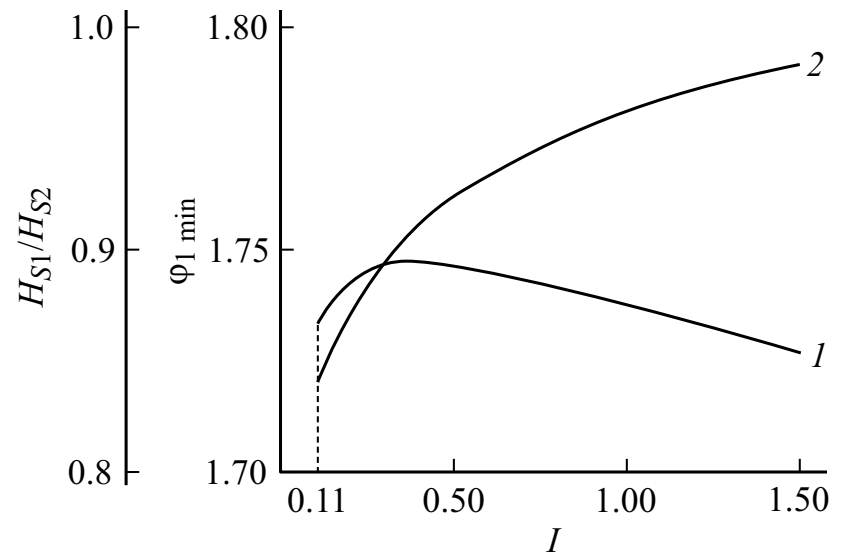

Рис. 4. Зависимости величин $\varphi_{1 \min }$ (кривая 1 ) и $H_{S 1} / H_{S 2}$ (кривая 2) от параметра $I$.

поле, равном $h_{S 1}$. Например, в табл. 2 в [5] приведены рассчитанные для $I=1$ и $I=0.1$ величины таких приращений, нормированные на „амплитуду“ флуктуации. Для использованного в настоящей работе значения $I=2.5$ расчет дает $0.321,0.048,0.006$. Умножим эти числа на какую-то малую „амплитуду“ , например, 0.001, добавим полученные значения к соответствующим $\varphi_{k}$ и $\xi_{k}$ и предоставим возможность „точке“ двигаться по „линии наибыстрейшего спуска“ , как это объяснялось выше. В смысле программирования это означает задать цикл, в каждом шаге которого новые значения $\varphi_{m}$ и $\xi_{m}$ при $1 \leq m \leq N$ вычисляются по формуле $\psi_{m}=\psi_{m}-\frac{\partial G}{\partial \psi_{m}} \delta$ с учетом (14)-(16). В конце концов, придем к конфигурации, в которой все производные $\partial G / \partial \psi_{i}$ обратятся в ноль, т.е. будут выполнены условия (1). Это и есть искомая равновесная конфигурация, отличающаяся от исходной мейсснеровской. Она является итогом первого этапа расчета. Начиная от нее, как говорилось выше, можно дать приращение $\Delta h$ магнитному полю, проследить переход системы в новое равновесное состояние и т.д. Это приращение может быть и отрицательным, что означает движение в сторону уменьшения внешнего магнитного поля. Дойдя при положительных $\Delta h$ до некоторого значения поля $h_{a}$, сменим знак $\Delta h$ и начнем отслеживать развитие ситуации при уменьшении поля. Далее, достигнув значения $-h_{a}$, снова начнем увеличивать поле. Таким способом будет исследован весь цикл.

Выполнение условий (1) будем проверять, вычисляя магнитное поле $h_{m}=\Phi_{m} / \Phi_{0}$ внутри $m$-й ячейки по формуле

$$
2 \pi h_{m}=-2 \pi h+I \sum_{i=1}^{m} \sin \psi_{i}
$$

Такая проверка показывает, что условия (1) с высокой точностью выполняются в конце как первого этапа, так и каждого цикла расчетов (при новом значении $h$ ) на втором этапе. 


\section{Результаты расчетов, их интерпретация и анализ}

Критическое значение параметра пиннинга $I_{C}$, вычисленное в работе [7], находится в диапазоне $0.7-0.8$. Для исследования было выбрано значение $I=2.5>I_{C}$, при котором $h_{S 1}=0.602$.

Компьютерные расчеты полностью подтвердили возможность использования предложенного алгоритма для расчета проникновения поля в среду. Действительно, постепенным увеличением значения $h$ от $h_{S 1}=0.602$ удается проследить постепенное изменение конфигурации токов, причем при каждом $h$ найденные конфигурации удовлетворяют условиям (1). Получающиеся решения имеют период вдоль границы, равный двум ячейкам (рис. 3). Будем далее называть конфигурацию шириной в две ячейки, периодическое повторение которой дает искомые токовые распределения, основой. Основа состоит из двух столбцов. При исследовании конфигурации основы оказывается удобной интерпретация ее как последовательности вихрей, проникших в образец. При этом анализ распределения скачков фазы [7] показывает, что центры вихрей находятся в тех ячейках, в которых $\psi_{m}$ (т.е. $\varphi_{m}$ или $\left.\xi_{m}\right)$ впервые (если двигаться из глубины) превосходят значения $2 \pi K+\pi$ $(K-$ целое число). Отсюда следует, что для трактовки картины как последовательности вихрей нужно каждый раз, как только $\psi_{m}$ превосходит $\pi$, вычитать из него целое число раз по $2 \pi$ так, чтобы все $\left|\psi_{m}\right|<\pi$. При этом количество вычтенных $2 \pi$ минус аналогичная величина на предыдущем разе даст значение флюксоида в соответствующей ячейке.

Характер вихревых последовательностей при увеличении магнитного поля для случая $I>I_{C}$ подробно исследовался в [7]. Настоящее исследование позволяет найти вид этих последовательностей при циклическом изменении внешнего поля.

В случае $I<I_{C}$ магнитное поле пронизывает все сечение образца, поэтому в работе [9] анализировалась магнитная индукция, равная отношению полного магнитного потока к площади сечения образца. В рассматриваемом случае этот подход непригоден, так как площадь сечения бесконечна, а поток конечен и сосредоточен в приграничной области. Поэтому будем исследовать зависимость от внешнего поля $h$ магнитного потока $\tilde{\Phi}$ через одну основу (две ячейки) от края вглубь, равного, согласно (10), $\tilde{\Phi} / \Phi_{0}=\left(\varphi_{1}+\xi_{1}\right) / 2 \pi$.

На рис. 5 изображена зависимость $2 \pi \tilde{\Phi} / \Phi_{0}$ от внешнего поля $2 \pi h$ для $I=2.5$. Участок АВС представляет собой основную кривую намагничивания, получаемую при монотонном увеличении внешнего поля от нуля. Буквами $D, D^{\prime}$ и $E, E^{\prime}$ обозначены точки разворота. В точках $F^{\prime}$ и $G^{\prime}$ распределения в точности совпадают с распределениями в точках $F$ и $G$, отличаясь лишь знаком.

На рис. 6 изображены основы, соответствующие основной кривой намагничивания при $I=2.5$ (в нижней

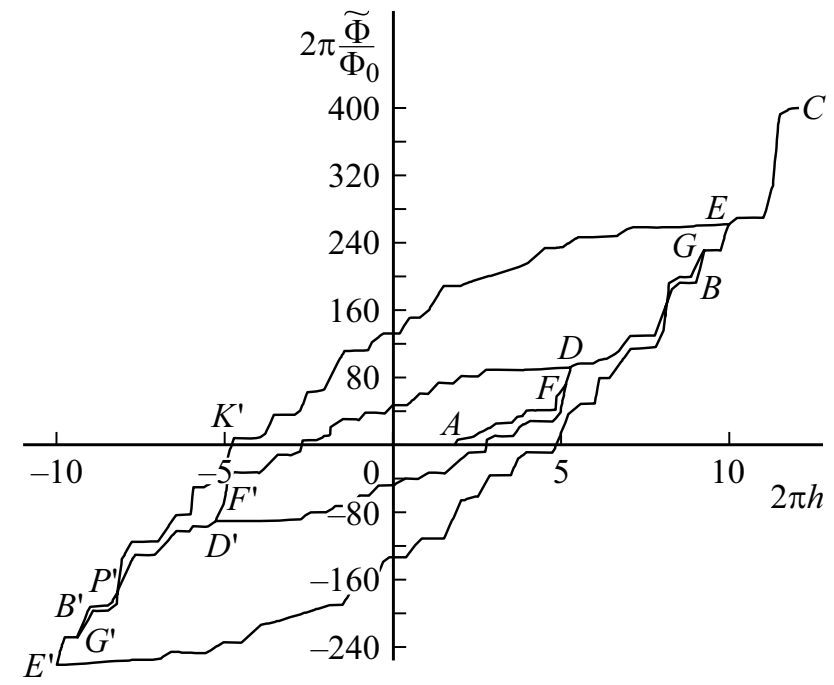

Рис. 5. Зависимость нормированного магнитного потока через два столбца основы от внешнего поля $h$ для $I=2.5$. В точках $F^{\prime}$ и $G^{\prime}$ распределения в точности совпадают с распределениями в точках $F$ и $G$, отличаясь лишь знаком.

строке указаны значения 2лh). Верхний ряд на рис. 6 соответствует границе образца. Ячейки с флюксоидом, равным 1 , обозначены крестами. В случае $2 \pi h=24$ цифры обозначают число вихрей в ячейке. Отметим, что в этой основе не показаны вихри ниже нижнего ряда, содержащие постепенно уменьшающееся до нуля количество квантов потока.

На рис. 7 приведены основы для обратного хода зависимости при развороте в точке $D\left(2 \pi h_{a}=10.5\right)$. Можно видеть, что по мере уменьшения внешнего поля некоторые вихри, поодиночке или парами, покидают основу. Причиной этого является взаимное отталкивание одинаково ориентированных вихрей. Внешнее поле, подобно внешней силе, сжимающей пружину, заставляло вихри войти в образец и удерживало их там. При уменьшении поля его влияние ослабевает, и отталкивание заставляет часть вихрей покинуть образец. Кроме того, вихри удерживаются внутри еще и за счет пиннинга. Поэтому при нулевом внешнем поле в образце остается значительное число вихрей. При некотором значении отрицательного внешнего поля внутрь образца начинают входить вихри противоположной ориентации. Это можно увидеть на рис. 7. Буквой „у“ обозначены вихри с флюксоидом, равным -1. При $2 \pi h=-3.72$ первый такой вихрь проникает в образец, при $2 \pi h=-3.8$ в правом столбце основы отрицательный вихрь аннигилирует с положительным (оба исчезают), а в левый столбец входит еще один отрицательный. При $2 \pi h=-5.0$ входит второй и т.д. Поскольку вихри противоположных ориентаций притягиваются друг к другу, а при достаточно малом расстоянии между ними пиннинг не может их удержать, они сливаются, что соответствует взаимному уничтожению (,аннигиляции“). Поэтому существование вихрей обеих ориентаций возможно только при значительном 


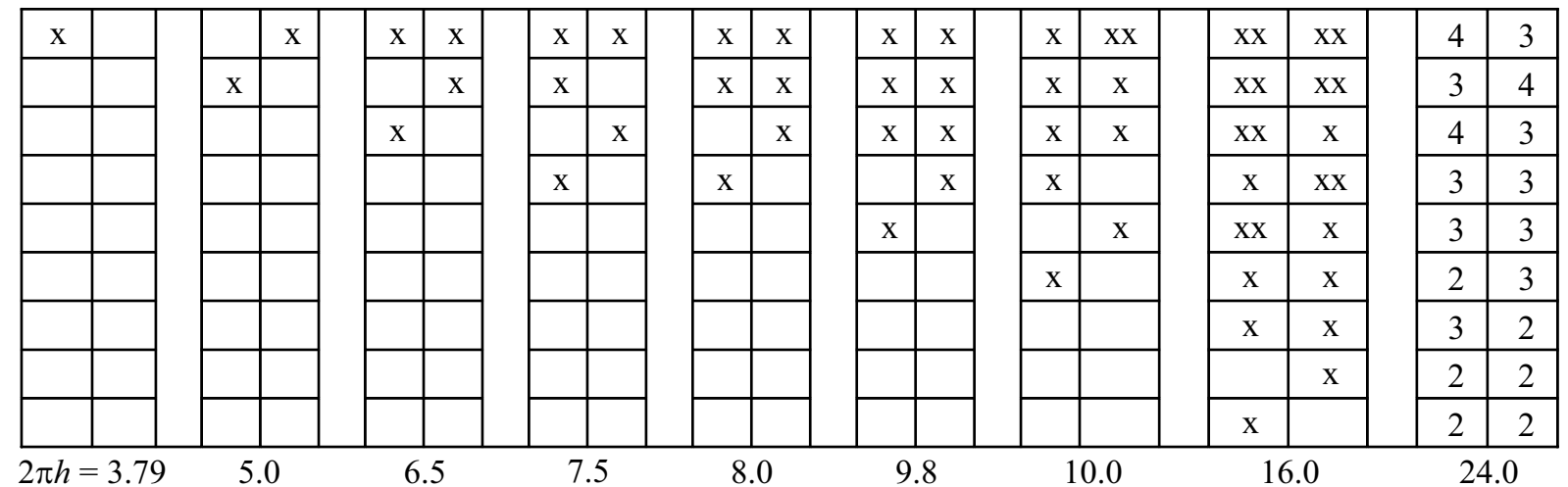

Рис. 6. Вихревые конфигурации основы, соответствующие основной кривой намагничивания для $I=2.5$ при различных значениях внешнего поля $h$ (указаны внизу).
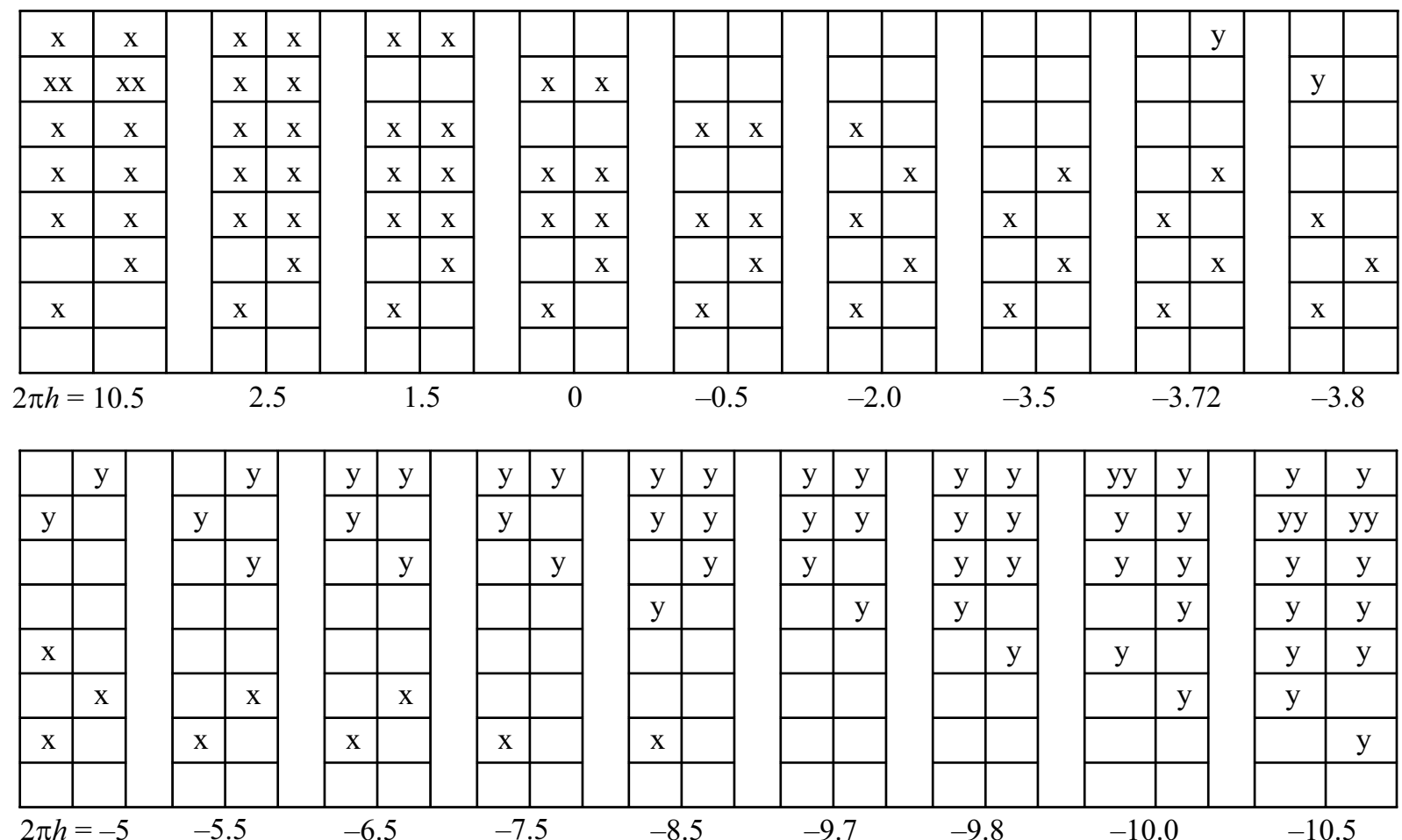

Рис. 7. Вихревые конфигурации основы для точек разворота $D$ и $D^{\prime}$ при различных значениях внешнего поля $h$ (указаны внизу).

расстоянии между ними. При $2 \pi h=-5.5$ отрицательный вихрь аннигилирует со следующим положительным и т.д.

Из рис. 7 видно, что проникновение в образец отрицательных вихрей начинается при меньшем по модулю значении внешнего поля $h=3.72 / 2 \pi=0.592$, чем $h_{S 1}=0.602$, при котором впервые начинают проникать положительные вихри. Это объясняется тем, что при $h=-0.592$ в глубине существует положительный вихрь, который своим притяжением способствует вхождению в образец первого отрицательного. Поэтому он входит при меньшем значении внешнего поля, чем первый вихрь на основной кривой, когда никаких вихрей внутри нет.
Сравнивая рис. 6 и 7, легко видеть, что, начиная с того момента, когда остаются только отрицательные вихри $(2 \pi h=-9.7$ на рис. 8$)$, последовательности отрицательных вихрей совпадают с последовательностями положительных из основной кривой (рис. 6). Точное совпадение распределений фаз происходит только в точке $F^{\prime}(2 \pi h=-10.0)$. В ней распределение $\varphi_{m}$ в точности совпадает с распределением в точке $F(2 \pi h=10.0)$, отличаясь лишь знаком. Это значит, что, начиная от точки $F^{\prime}$, участок кривой $F^{\prime} D^{\prime} F$ на рис. 5 будет симметричен участку $F D F^{\prime}$ и завершится в точке $F$.

Те же выводы можно сделать и из рассмотрения большой петли на рис. 5 , соответствующей развороту в 


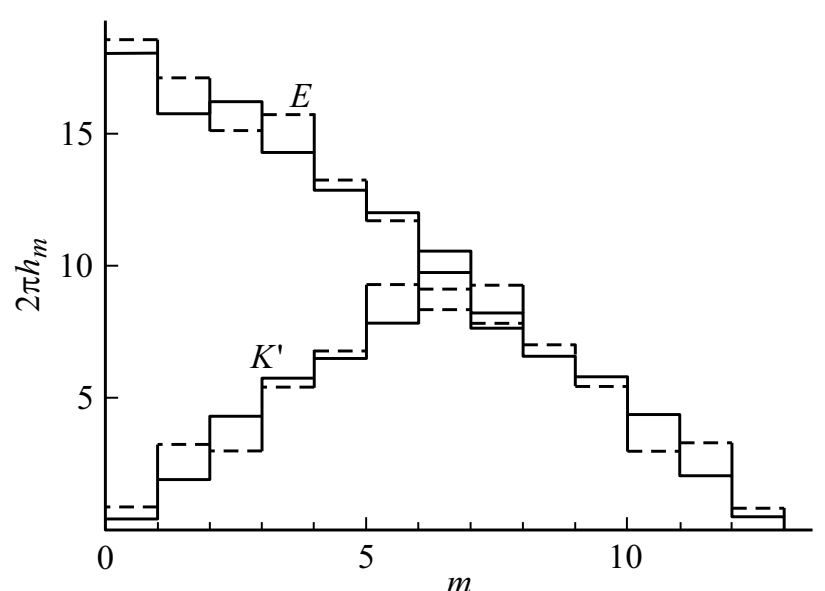

Рис. 8. Зависимости магнитного поля $h_{m}$ в ячейке от расстояния $m$ до границы (в количестве ячеек) в двух столбцах основы при $I=2.5$ для двух значений магнитного поля (точки $E$ $(2 \pi h=20)$ и $K^{\prime}(2 \pi h=0)$ на рис. 5$)$.

точке $E\left(2 \pi h_{a}=20.0\right)$. В этом случае полное совпадение наступает в точке $G^{\prime}\left(2 \pi h_{a}=-18.5\right)$. Обращает на себя внимание факт пересечения в точке $P^{\prime}(2 \pi h \approx-16.3)$ этой петли с участком основной кривой $F^{\prime} D^{\prime} B^{\prime} G^{\prime} E^{\prime}$. Это можно объяснить тем, что при $2 \pi h=-16$ в глубине образца за несколькими отрицательными вихрями остается еще вихрь другого знака (положительного), который уменьшает силу, препятствующую вхождению нового вихря. Поэтому новый вихрь входит при меньшем значении внешнего поля, чем на основной кривой, где такого вихря нет, и при $2 \pi h=-16.5$ конфигурация содержит на один вихрь больше, чем при $2 \pi h=16.5$ на основной кривой. Начиная с точки $G^{\prime}(2 \pi h=-18.5)$ это различие исчезает.

Изложенные выше соображения объясняют, почему уже после первого оборота петля гистерезиса замыкается сама на себя. При обратном ходе проникновение отрицательных вихрей облегчается за счет существования в глубине оставшихся положительных. Поэтому еще до разворота (при $\left.-h_{a}\right)$ положительные вихри в глубине исчезают (в результате аннигиляции с отрицательными), и крайние участки петли оказываются на основной кривой. После этого все повторяется сначала.

Формулы (17) или (1) позволяют найти магнитное поле $h_{m}$ в любой ячейке. В [7] показано, что при монотонном увеличении внешнего поля от нуля поле в образце убывает с расстоянием от границы внутрь практически линейно. Крутизна убывания (усредненное изменение величины $h_{m}$ при увеличении $m$ на 1 ) определяется значением параметра пиннинга и представляет собой рациональную дробь. При $I=2.5$ эта крутизна, согласно [7], равняется $1 / 4$.

Рассмотрим рельеф магнитного поля у границы образца для обратного хода внешнего поля, т.е. при его уменьшении. На рис. 8 сплошной и штриховой кривыми изображены зависимости магнитного поля $h_{m}$ в ячейке от расстояния $m$ до границы (в количестве ячеек) в двух столбцах при $I=2.5$ для двух значений магнитного поля (точки $E(2 \pi h=20)$ и $K^{\prime}(2 \pi h=0)$ на рис. 5). Легко видеть, что значения магнитного поля в соседних ячейках двух столбцов основы различаются, на кривых есть отклонения от линейного хода, в некоторых точках наблюдается даже нарушение монотонности убывания.

На рис. 9 изображены зависимости от расстояния $m$ магнитного поля $h_{m}$, усредненного по двум столбцам (среднее арифметическое кривых рис. 8) для различных значений магнитного поля из обратного хода большой петли (участок $E E^{\prime}$ на рис. 5). Магнитное поле у поверхности образца снаружи равно внешнему. Поэтому значения $2 \pi h$ указаны слева от оси ординат. Осцилляции на кривых рис. 9 заметно меньше, чем на рис. 8. Нарушения монотонности отсутствуют.

Из рис. 9 видно, что усредненный рельеф магнитного поля в образце представляет собой два линейных участка - возрастающий и спадающий, причем крутизна на обоих участках одинакова по модулю и равна $1 / 4$, что совпадает с результатами [7]. С убыванием внешнего поля точка излома все дальше сдвигается в глубину образца, пока не исчезает совсем, когда график начинает представлять собой участок только одной прямой.

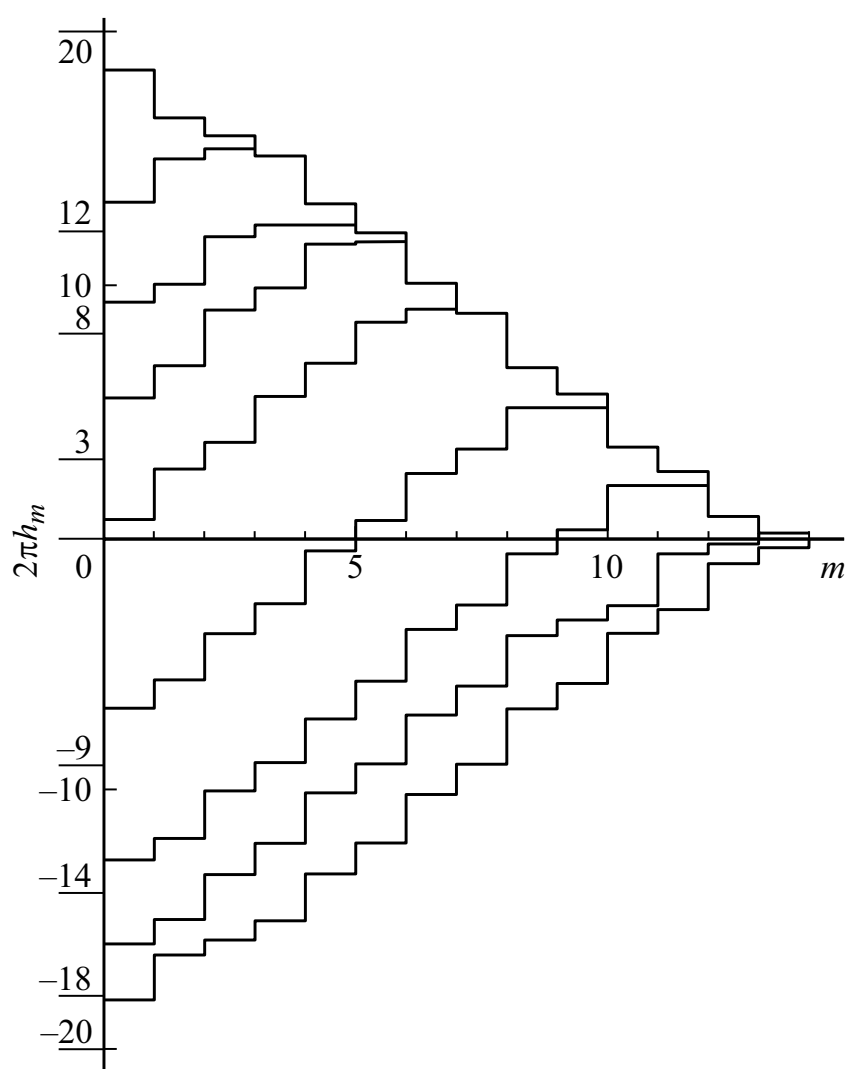

Рис. 9. Зависимости от расстояния до границы $m$ магнитного поля $h_{m}$ в ячейке, усредненного по двум столбцам, для различных значений внешнего магнитного поля $h$ из обратного хода большой петли (значения $2 \pi h$ указаны слева от оси ординат). 


\section{Заключение}

На базе подхода, основанного на анализе непрерывного видоизменения конфигурации, протекающего в направлении уменьшения потенциала Гиббса, рассчитана кривая намагничивания трехмерной упорядоченной джозефсоновской среды при циклическом изменении внешнего магнитного поля для немалых значений параметра пиннинга. Показано, что крайние области петли гистерезиса всегда лежат на основной кривой намагничивания.

Исследованы изменения вихревых последовательностей, а также профиля магнитного поля в образце при циклическом изменении внешнего поля. Показано, что рельеф магнитного поля в образце состоит из двух линейных участков - возрастающего и убывающего с одинаковыми по модулю значениями крутизны.

\section{Список литературы}

[1] Галайко В.П. // ЖЭТФ. 1966. Т. 50. С. 717.

[2] Kramer L. // Phys. Rev. 1968. Vol. 170. P. 475.

[3] Zelikman M.A. // Supercond. Sci. Technol. 1997. Vol. 10. N 7. P. 469-474.

[4] Zelikman M.A. // Supercond. Sci. Technol. 1997. Vol. 10. N 11. P. 795-800.

[5] Зеликман М.А. // ЖТФ. 2008. Т. 78. Вып. 8. С. 14-21.

[6] Зеликман М.А. // ЖТФ. 2009. Т. 79. Вып. 2. С. 36-42.

[7] Зеликман М.А. // ЖТФ. 2009. Т. 79. Вып. 9. С. 47-57.

[8] Зеликман М.А. // ЖТФ. 2010. Т. 80. Вып. 3. С. 21-30.

[9] Зеликман М.А. // ЖТФ. 2017. Т. 87. Вып. 9. С. 1346-1353. 